\section{Case Reports in Oncology}

\title{
Postsurgical Pyoderma Gangrenosum in a Breast Cancer Patient: A Case Report and Literature Review
}

\author{
Grazia Maria Vernaci ${ }^{a}$, \\ Tania Saibene $^{d}$ Maria Cristina Montesco ${ }^{e}$ Enrico Orvieto ${ }^{f}$ \\ Annamaria Cattelang ${ }^{a}$ Cristina Ghiotto $^{b}$ Federica Miglietta ${ }^{a}$ b \\ Valentina Guarneria, b Matteo Cagol ${ }^{d}$
}

\begin{abstract}
aDepartment of Surgery, Oncology and Gastroenterology, University of Padua, Padua,

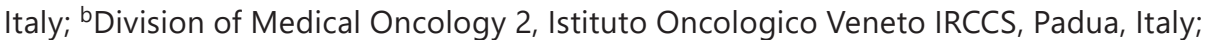
${ }^{C}$ Anaesthesiology Unit, Istituto Oncologico Veneto IRCCS, Padua, Italy; ${ }^{d}$ Breast Surgery Unit, Istituto Oncologico Veneto IRCCS, Padua, Italy; ${ }^{\mathrm{e}}$ Anatomy and Histology Unit, Istituto

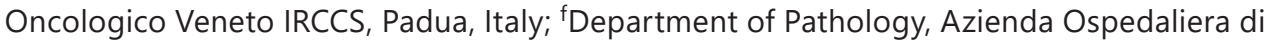
Rovigo, Rovigo, Italy; 9Unit of Infectious Disease, Department of Internal Medicine, Azienda Ospedaliera Universitaria di Padova, Padua, Italy
\end{abstract}

\section{Keywords}

Pyoderma gangrenosum · Solid tumors · Breast cancer · Pathergy

\begin{abstract}
Pyoderma gangrenosum is a rare skin necrotizing disease that can arise on a site of surgical trauma. Its pathogenesis has recently been related to dysregulation of the immune system, with inflammatory bowel disease representing the most commonly underlying systemic conditions. Several authors have also reported an association with solid malignancies (especially gastrointestinal and breast cancer). We describe the case of a 39-year-old patient diagnosed with a locally advanced, triple-negative breast cancer who developed a pyoderma gangrenosum on the surgical wound after a CVC implant with systemic complications. As the diagnosis and management of postsurgical pyoderma gangrenosum can be challenging for clinicians, underlying conditions as autoimmune disease and solid tumors have to be considered in order to guide treatment.




\section{Introduction}

Pyoderma gangrenosum (PG) is a rare inflammatory dermatosis, characterized by necrotizing skin lesions. Ulcerative phenotype represents the most common clinical presentation, typically occurring as painful cutaneous erosions with peripheral expansion and central degeneration [1]. Although lesions could arise on intact skin, PG often develop at a site of a preceding trauma. This phenomenon, known as pathergy, may pose a diagnostic dilemma, since PG can mimic surgical site infection with no pathognomonic characteristics.

Although no formal guidelines exist, general treatment principles can be delineated based on available evidence. Clinical management includes local treatment and systemic therapy with corticosteroids and immunosuppressant. In the absence of controlled trials, second-line treatment of PG can rely on some experimental options that proved to be worthwhile, namely biological immunomodulatory as the anti-TNF $\alpha$ infliximab and intravenous (i.v.) immunoglobulins, conventional immunosuppressants (as mycophenolate mofetil), or alkylating agents, such as cyclophosphamide, which has recently been suggested as a promising corticosteroid-sparing anti-PG therapy [2].

Importantly, in case an underlying systemic disease is detected, treatment of such condition can actually result in PG remission.

Given the likelihood of pathergy, the role of surgery in PG management is controversial and should be restricted to selected cases. However, when surgery cannot be avoided or delayed, it requires adequate systemic perioperative coverage [2].

Despite the above-mentioned role of pathergy, the exact pathogenesis of PG is still unclear. An association with autoimmune disease (especially inflammatory bowel disease (IBD) and rheumatoid arthritis) has been demonstrated. Evidence of a possible association between PG and solid tumors has also been reported.

Here, we present the case of a young woman diagnosed with breast cancer (BC) and a postsurgical PG.

\section{Case Presentation}

A 39-year-old woman was diagnosed with a palpable mass on her right breast in March 2017. Her past medical history showed a Hodgkin lymphoma in 2001, treated with chemotherapy ( 6 courses of ABVD) and mantle field radiation. Her medical history also showed an inflammatory colitis since she was adolescent, with the last flare in 2016, treated with mesalazine. No specific therapy was ongoing at the time the patient presented at our Institute.

Concerning her family history, her younger sister died from a metastatic myxoid liposarcoma; no cases of breast or ovarian tumors were found.

Radiological examination of the breast showed a nodular area of about $30 \mathrm{~mm}$. Pathological examination confirmed an invasive ductal carcinoma, G3, with triple-negative phenotype and MIB1 85\%. A staging CT scan of the thorax and abdomen was negative for distant metastasis.

After multidisciplinary discussion, neoadjuvant chemotherapy with paclitaxel $80 \mathrm{mg} / \mathrm{m}^{2}$ plus carboplatin AUC2 d1-8-15 q28 for 4 cycles was planned (no anthracyclines due to previous exposure). Patient completed the first cycle on April 19,2017, with no hematological toxicity of any grade. On April 21, 2017, 3 days after receiving the third chemotherapy administration, because of the poor venous heritage, patient underwent port-à-cath insertion.

On May 2, 2017, the patient presented to emergency room with a temperature of $38.8^{\circ} \mathrm{C}$ and normal vital signs; the skin overlying PORT showed signs of subcutaneous cellulitis and colliquative necrosis. Blood works found elevated white blood cell count $\left(21,000 / \mathrm{mm}^{3}\right)$, with 


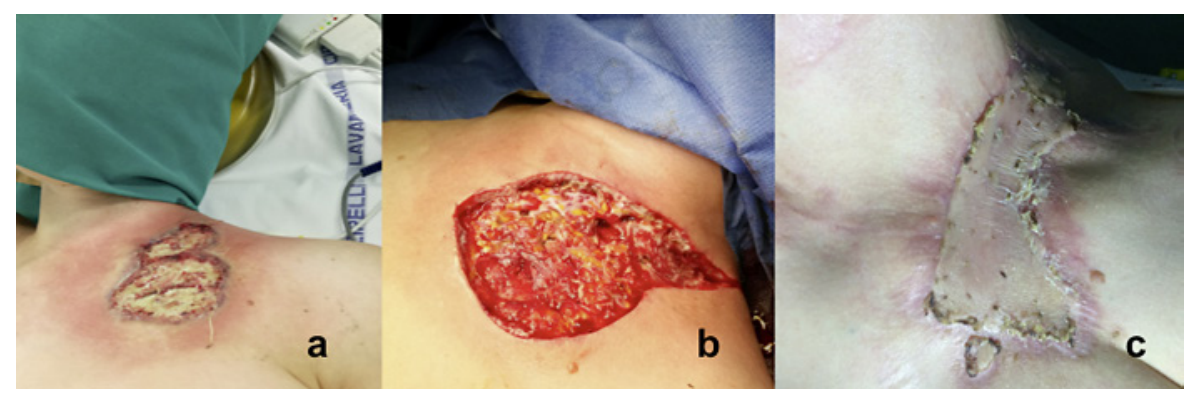

Fig. 1. Skin lesion course. a At the beginning. b After necrosectomy. c After skin graft.

neutrophilia $\left(16,470 / \mathrm{mm}^{3}\right)$ and elevated C-reactive protein $(250 \mathrm{mg} / \mathrm{L})$. A broad-spectrum i.v. antibiotic therapy with piperacillin/tazobactam and daptomycin was started, and the patient underwent PORT rimotion and necrosectomy, with defervescence and improvement in subcutaneous cellulitis and blood works.

On May 6, 2017, because of new febrile seizure with WBC rise $\left(20,510 / \mathrm{mm}^{3}\right)$ and worsening of skin lesion, the patient underwent a second necrosectomy. Peripheral blood cultures and skin plug were negative for infection; i.v. catheter tip showed positivity for Klebsiella pneumoniae sensitive to both meropenem and levofloxacin; therefore, antibiotic therapy was modified accordingly. A chest/abdomen CT scan showed mediastinitis and bilateral pleural effusion with left pulmonary atelectasis. Patient was transferred to the Thoracic Surgery Unit and underwent left thoracoscopy with pleural and mediastinal drainage. Then she was admitted to the Intensive Care Unit for observation and support. The postoperative course was characterized by sepsis, which required broad-spectrum antibiotic and antifungal therapy, hemodynamic support and non-invasive ventilation.

Specimens of skin and subcutaneous and muscular tissue from necrosectomy were analyzed, with evidence of an intensive inflammatory infiltrate including mainly neutrophils; the differential diagnosis included PG.

In our patient, the presence of concomitant aggressive triple-negative $\mathrm{BC}$ precluded the use of high-dose corticosteroids, thus limiting our treatment options. Systemic methylprednisolone $20 \mathrm{mg}$ /day and topical cyclosporine were started. Seriate chest X-ray and CT scan showed progressive resolution of mediastinitis and pleural effusion (suppl. Fig. 1; for all suppl. material, see www.karger.com/doi/10.1159/000509745). Wound improvement with scar was evident (Fig. 1). Blood works also indicated progressive normalization of blood count and flogosis index.

After hemodynamic and clinical stabilization, the patient underwent breast ultrasound, which showed no change in the dimension of the lump. After multidisciplinary meeting, on May 29, 2017, the patient underwent right mastectomy and axillary dissection. Breast surgical wound healing was regular. Pathology assessment revealed fibroelastosis and chronic inflammation, with isolated neoplastic cells for maximum $2 \mathrm{~mm}$ extension; 8 axillary nodes were negative. A restaging brain/chest/abdomen CT was negative for distant metastasis. The BRCA and p53 mutation tests were negative.

On June 16, 2017, the patient was released from the hospital. In order to allow the PORT wound healing, she underwent an autologous skin graft, with no further complications. As for multidisciplinary assessment, after a PICC implant, the patient resumed chemotherapy with carboplatin and paclitaxel with dose reduction. In November 2017, she completed her fourth and last cycle, with good tolerance, and started the follow-up.

\section{Karger ${ }^{\prime}=$}


Fig. 2. Tumor infiltrating lymphocytes. No infiltration was observed on the pretreatment sample (a), while a moderate infiltrate was present in the surgical sample (b).

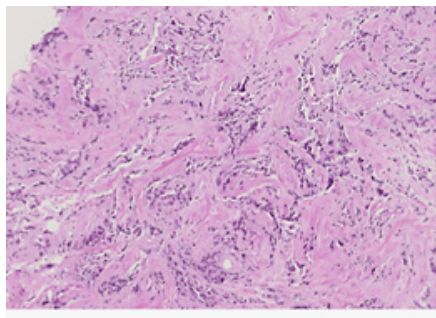

a

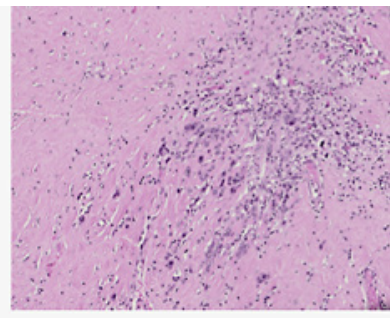

b

\section{Discussion and Conclusions}

Due to the lack of pathognomonic features, the diagnosis of postsurgical PG is made clinically, by exclusion - pathergy may represent a diagnostic dilemma for surgeons, and a timely diagnosis is crucial [3].

Although the exact pathogenesis remains unclear, deregulation of the immune system has been proposed to be involved. Interestingly, in more than half of the patients, an underlying systemic disease that can both precede and/or follow PG is detected. IBD, such as Crohn's disease and ulcerative colitis, represent the most common association [2]. In addition, PG has been associated with solid malignancies (especially gastrointestinal and $\mathrm{BC}$ ), but available evidence is mostly restricted to case reports [4]. In particular, it has been reported that PG can herald a recurrence of previous solid malignancy, and it can occur postoperatively after tumor-related surgery [3-9].

In this context, there have been several reports of tumor and PG diagnoses occurring within 1 year of each other with no other active underlying systemic conditions $[4-6,8]$ (suppl. Table 1). In a proportion of these cases, pathergy was identified as the trigger factor $[4,5,8]$. Interestingly, several authors reported PG improvement with the combination of PG-specific and anti-tumor therapy [4, 7] or even with anti-tumor treatment alone, further suggesting an entanglement between cancer and PG. Indeed, despite the lack of robust evidence, it can be speculated that aberrant immune surveillance mechanisms could represent a possible biological explanation for this link, as already suggested for paraneoplastic conditions related to immune deregulation mediated by cancer cells [4].

Our patient underwent mastectomy with no complications, and the pathological assessment revealed a very good response, which was surprising considering that she had only received 1 course of chemotherapy.

If cancer-mediated immune deregulation could trigger PG, on the other hand, we could hypothesize an association in the opposite direction, where the immune activation underlying PG could foster an anti-tumor immune response (i.e., by immune activation against cross-reactive antigens expressed by tumor cells?). Of course, this is a speculation, and we do not have any evidence in support of this. However, with a descriptive purpose, we looked at the immune infiltrate on tumor samples collected at diagnosis and at surgery. No lymphocytic infiltration was detected on the pre-treatment sample, whereas a moderate infiltrate was observed in the surgical sample as shown in Figure 2.

Postsurgical PG represents a challenging diagnosis and should be suspected in the presence of a skin lesion that fails to recover despite surgical debridement and systemic treatment. Underlying conditions such as autoimmune disorders and even solid tumors have to be considered, since the treatment of associated diseases can influence PG healing. 


\section{Case Reports in Oncology}

\begin{tabular}{l|l}
\hline Case Rep Oncol 2021;14:160-164 \\
\hline DOI: 10.1159/000509745 & $\begin{array}{l}\text { @ 2021 The Author(s). Published by S. Karger AG, Basel } \\
\text { www.karger.com/cro }\end{array}$ \\
\hline
\end{tabular}

Vernaci et al.: Pyoderma Gangrenosum in a Breast Cancer Patient

\section{Statement of Ethics}

The subject has given her written informed consent to publish this case report and images.

\section{Conflict of Interest Statement}

The authors declare that they have no conflict of interest.

\section{Funding Sources}

None.

\section{Author Contributions}

G.M.V., M.V.D., V.G., M.C., T.S., A.M.C. and M.M. followed the patient; E.O. and M.C.M. performed pathological assessment; G.M.V. and F.M. wrote the manuscript; M.V.D. and F.M. evaluated TILs; M.V.D. and V.G. conceived the study and gave the final approval of the version to be published. All authors revised the work critically and approved the final version of the manuscript. All authors agreed to be accountable for all aspects regarding the manuscript.

\section{References}

1 Ruocco E, Sangiuliano S, Gravina AG, Miranda A, Nicoletti G. Pyoderma gangrenosum: an updated review. J Eur Acad Dermatol Venereol. 2009;23(9):1008-17.

2 Ahronowitz I, Harp J, Shinkai K. Etiology and management of pyoderma gangrenosum: a comprehensive review. Am J Clin Dermatol. 2012;13(3):191-211.

3 Pereira N, Brites MM, Gonçalo M, Tellechea O, Figueiredo A. Pyoderma gangrenosum -- a review of 24 cases observed over 10 years. Int J Dermatol. 2013;52(8):938-45.

4 Shahi V, Wetter DA. Pyoderma gangrenosum associated with solid organ malignancies. Int J Dermatol. 2015; 54(9):e351-7.

5 Patel DK, Locke M, Jarrett P. Pyoderma gangrenosum with pathergy: a potentially significant complication following breast reconstruction. J Plast Reconstr Aesthet Surg. 2017;70(7):884-92.

6 Prystowsky JH, Kahn SN, Lazarus GS. Present status of pyoderma gangrenosum. Review of 21 cases. Arch Dermatol. 1989;125(1):57-64.

7 Duchnowska R, Ziajka E, Góralska A, Grala B. Recurrent pyoderma gangrenosum precipitated by breast cancer: a case report and review of the literature. J Med Case Rep. 2014;8:226-1947-8-226.

8 Mori T, Hiraiwa T, Ohtsuka M, Yamamoto T. Late-onset pyoderma gangrenosum following surgical operation in a patient with malignant melanoma. J Dermatol. 2015;42(6):650-1.

9 Jockenhofer F, Herberger K, Schaller J, Hohaus KC, Stoffels-Weindorf M, Ghazal PA, et al. Tricenter analysis of cofactors and comorbidity in patients with pyoderma gangrenosum. J Dtsch Dermatol Ges. 2016;14:1023-30.

\section{Karger'}

